\title{
Hydrophobic Proteome Analysis of Triple Negative and Hormone-Receptor-Positive-Her2-Negative Breast Cancer by Mass Spectrometer
}

\author{
Ming Lu • Stephen A. Whelan • Jianbo He • \\ Romaine E. Saxton • Kym F. Faull • \\ Julian P. Whitelegge • Helena R. Chang \\ Published online: 7 September 2010 \\ (C) The Author(s) 2010. This article is published with open access at Springerlink.com
}

\begin{abstract}
Introduction It is widely believed that discovery of specific, sensitive, and reliable tumor biomarkers can improve the treatment of cancer. Currently, there are no obvious targets that can be used in treating triple-negative breast cancer (TNBC). Methods To better understand TNBC and find potential biomarkers for targeted treatment, we combined a novel hydrophobic fractionation protocol with mass spectrometry LTQ-orbitrap to explore and compare the hydrophobic subproteome of TNBC with another subtype of breast cancer, hormone-receptor-positive-Her2-negative breast cancer (non-TNBC).

Results Hydrophobic sub-proteome of breast cancer is rich in membrane proteins. Hundreds of proteins with various defined key cellular functions were identified from TNBC and nonTNBC tumors. In this study, protein profiles of TNBC and non-TNBC were systematically examined, compared, and
\end{abstract}

M. Lu $\cdot$ S. A. Whelan J J. He $\cdot$ R. E. Saxton

Department of Surgery,

David Geffen School of Medicine at UCLA,

Los Angeles, CA, USA

H. R. Chang $(\bowtie)$

Revlon/UCLA Breast Center, Department of Surgery,

David Geffen School of Medicine at UCLA,

200 UCLA Medical Plaza, Suite B265-1,

Los Angeles, CA 90095-7028, USA

e-mail: hchang@mednet.ucla.edu

K. F. Faull $\cdot$ J. P. Whitelegge

The Pasarow Mass Spectrometry Laboratory, Department of

Psychiatry \& Biobehavioral Sciences and the Neuropsychiatric

Semel Institute for Neuroscience and Human Behavior,

David Geffen School of Medicine at UCLA,

Los Angeles, CA, USA validated. We have found that nine keratins are downregulated and several heat shock proteins are up-regulated in TNBC tissues. Our study may provide insights of molecules that are responsible for the aggressiveness of TNBC.

Conclusion The initial results obtained using a combination of hydrophobic fractionation and nano-LC mass spectrometry analysis of these proteins appear promising in the discovery of potential cancer biomarkers and bio-signatures. When sufficiently refined, this approach may prove useful in improving breast cancer treatment.

Keywords Hydrophobic fractionation - Cancer biomarker. Mass spectrometry - Triple-negative breast cancer.

Hormone-receptor-positive-Her2-negative breast cancer

\section{Abbreviations}

$\begin{array}{ll}\text { ER } & \text { Estrogen receptors } \\ \text { Her2 } & \text { Human epidermal growth factor receptor } 2 \\ \text { MHC } & \text { Major histocompatibility complex } \\ \text { PR } & \text { Progesterone receptors } \\ \text { TNBC } & \text { Triple-negative breast cancer }\end{array}$

\section{Introduction}

Breast cancer is the most common cancer in women, the leading cause of death among young women aged 15-54, and the second most common cause of cancer death in American women [1]. Breast cancer is heterogeneous molecularly, histologically, and clinically. As a result, different types of breast cancer require special treatment considerations. Chemical ligands such as hormones, when bound to receptors, lead to a chain of changes in the cell. 
Various receptors can be found on the cell surface, in the cytoplasm, and in the nucleus, which determine the biology and clinical behaviors of different types of breast cancer. In breast cancer, estrogen receptor (ER), progesterone receptor (PR), and human epidermal growth factor receptor 2 (Her2/neu) are the three biomarkers used clinically to guide treatment. Breast cancer with negative expression of the three biomarkers is known as triplenegative breast cancer (TNBC). TNBC accounts for approximately $15 \%$ of all invasive breast cancers $[2,3]$. Recent gene analysis studies suggest that TNBC arises from basal cells of the mammary epithelium [4-7]. TNBCs occur more frequently in African-American women, young women, and women with the BRCA1 mutations $[3,8,9]$. TNBC represents an aggressive type of breast cancer with early relapse and poor survival rate [10]. Unfortunately, chemotherapy remains the only available systemic treatment, as there have been no effective target therapies developed for TNBC.

Hormone receptor-positive Her2-negative breast cancer represents the other end of the disease spectrum. Genomic analysis classified them as luminal type A breast cancer [11-13], which not only carry a better prognosis but are also extremely susceptible to hormone therapy.

In this study, we compared the hydrophobic subproteome of the two types of breast cancer: TNBC and $\mathrm{ER}+\mathrm{PR}+\mathrm{Her} 2-$ breast cancer. We wish to identify the differentially expressed hydrophobic sub-proteome of TNBC, which may distinguish it from the ER+PR+Her2breast cancer and provide further molecular insights of its aggressive biology.

Proteomics has been employed recently to identify new disease-related biomarkers for cancer diagnosis and development of targeted treatment [14-19]. Since tumor/cancer specimens are rich in cancer-related proteins and are directly relevant to human disease, they were selected to study the bio-signature of breast cancer.

A common strategy used in proteomics research is to enrich a target set of proteins in order to identify the lower abundance proteins that are important in cancer biology. Many fractionation methods have been explored, including isolation/enrichment of the membrane sub-proteome, such as membrane glycoproteins [17], which may serve as a key area to discover therapeutic and diagnostic targets. In this study, we focused on membrane proteins as it accounts for approximately one third of all open reading frames of sequenced human genomes encoding the polytopic transmembrane proteins [20, 21]. Despite their critical biological significance, membrane proteins remain under-studied due to their poor solubility, making separation and mass analysis difficult $[22,23]$. Previously, our data demonstrated that more membrane proteins were found in the hydrophobic fraction of the tumor cells than the conventional membrane proteins prepared by the centrifugation method [19]. Whitelegge et al. [24-28] have also shown that not only cell membrane proteins with a variety of functions, but also sub-cellular organelle membrane proteins and acylated nonmembrane proteins were recovered in the hydrophobic subproteome. We therefore chose membrane proteins enriched by hydrophobic fractionation method to study a panel of 20 breast cancer samples.

Hydrophobicity is a common feature of many cellular proteins, especially those residing within or associated with lipid bi-layer membranes. Since membrane proteins play critical roles within cells and endow cancer cells with many of their unique properties, a strategy that enriches this class of proteins may prove to be useful. While integral membrane proteins can be predicted from their primary sequences, association of other globular proteins with membranes can be challenging if not impossible to predict. Choice of hydrophobicity as a property for enrichment is novel and offers the chance of finding "biomarker events" that result in gain or loss of membrane association. In this study, hydrophobic sub-proteome of breast cancer was analyzed by combining LTQ-Orbitrap mass spectrometry with several computational methods to identify a cohort of moderate abundance proteins including several candidate biomarkers related to malignancy.

\section{Material and Methods}

\section{Human Samples}

Breast specimens were collected prospectively from participants of an IRB-approved clinical study for discovery of cancer biomarkers. Breast tissue was collected immediately after needle biopsy or surgical removal according to the standard operating procedures established for tissue and bio-specimen collection. The collected specimen was delivered on ice to the Tissue Bank, where it was divided into three parts: one in OCT embedding medium for frozen tissue specimens (Tissue-Tekâ) and two directly frozen in liquid nitrogen. All specimens were stored frozen at $-80^{\circ} \mathrm{C}$.

Ten cases of each of the TNBC and ER+PR+Her2(non-TNBC) breast cancer tissues were compared. The clinical histo-pathological information of the 20 cases is summarized in Table 1.

\section{Protein Extraction of Tumor Tissues}

After removing the visible fat, breast tumors were rinsed in cold PBS and homogenized in cold lysing buffer $(150 \mathrm{mM}$ $\mathrm{NaCl}, 50 \mathrm{mM}$ Tris-Cl, $2 \mathrm{mM}$ EDTA, $1 \mathrm{mM}$ sodium orthovanadate, containing $1 \%$ Triton X-100). The homogenizer was immersed in slushy ice during 30 slow passes. The 
Table 1 Clinical characteristics and pathological features of tumors used in this study

\begin{tabular}{lllll}
\hline Case no. & Age & Ethnicity & Clinical stage & ER/PR/Her2 \\
\hline 1 & 61 & White & T3N0 & $(-/-/-)$ TNBC \\
2 & 45 & Hispanic & T3N0 & $(-/-/-)$ TNBC \\
3 & 55 & Hispanic & T3N0 & $(-/-/-)$ TNBC \\
4 & Hispanic & T3N0 & $(-/-/)$ TNBC \\
5 & 54 & Asian & T3N1 & $(-/-)$ TNBC \\
6 & 40 & White & T3N1 & $(-/-/-)$ TNBC \\
7 & 44 & Asian & T4N0 & $(-/-/-)$ TNBC \\
8 & 84 & White & T2N0 & $(-/-/-)$ TNBC \\
9 & 38 & White & T2N0 & $(-/-/-)$ TNBC \\
10 & 52 & White & T2N0 & $(+/+/-)$ non-TNBC \\
Age average & 52 & & & $(+/+/-)$ non-TNBC \\
1 & 52.5 & Black & T3N1 & $(+/+/-)$ non-TNBC \\
2 & 57 & White & T3N0 & $(+/+/-)$ non-TNBC \\
3 & 45 & White & T3N3 & $(+/+/-)$ non-TNBC \\
4 & 65 & White & T4N2 & $(+/+/-)$ non-TNBC \\
5 & 49 & Hispanic & T4N1 & $(+/+/-)$ non-TNBC \\
6 & 39 & White & T3N0 & $(+/+/-)$ non-TNBC \\
7 & 48 & White & T3N0 & $(+/+/-)$ non-TNBC \\
8 & 62 & White & T2N0 & $(+/+/-)$ non-TNBC \\
9 & 48 & Asian & T2N0 & T3N1 \\
10 & 43 & White & &
\end{tabular}

homogenate of each specimen was centrifuged at $12,000 \times \mathrm{g}$ for $10 \mathrm{~min}$ at $4^{\circ} \mathrm{C}$ to remove debris. Supernatants were collected at $4^{\circ} \mathrm{C}$, and protein concentration of each sample was determined by Bradford assays before being frozen in liquid nitrogen for storage at $-80^{\circ} \mathrm{C}$.

\section{Hydrophobic Fractionation}

We have successfully developed a hydrophobic fractionation method to enrich membrane proteins [19]. We have compared three different methods to enrich membrane hydrophobic proteins by (1) traditional centrifugation method, (2) hydrophobic column, (3) disposable spin cartridge. We found the hydrophobic column identified many more membrane proteins than the traditional centrifugation method. However, the hydrophobic column suffered from a serious pressure build-up problem, which limited its use in handling multiple samples. The findings led to the development of disposable spin cartridge packed with beads of polystyrene-divinylbenzene polymers, which are the same as those used for commercially made hydrophobic column. The beads were packed into a single-use disposable spin cartridge to the same volume as the hydrophobic column. The proteins identified by the hydrophobic column and the disposable spin cartridge showed an overlap greater than $95 \%$. The disposable spin cartridge has become the method of choice for hydrophobic fractionation in our laboratory because of its proven functionality, no pressure- build up, low cost, and zero sample-to-sample cross contamination.

Briefly, each cartridge was activated with two sequential methanol rinses, followed by washing with three sequential rinses of water/acetonitrile/TFA (95/5/0.1, all by vol.). Specimens of 1-mg cell lysates were loaded into the cartridges. The cartridges were spun for $1 \mathrm{~min}$ at $110 \times g$ to remove the soluble proteins, salts, and polar solutes such as DNA with five sequential barrel washes using the following solvents:

1. Water/acetonitrile/isopropanol/TFA (90/05/05/0.1, all by vol.)

2. Water/acetonitrile/isopropanol/TFA (70/15/15/0.1, all by vol.)

3. Water/acetonitrile/isopropanol/TFA (50/25/25/0.1, all by vol.)

4. Water/acetonitrile/isopropanol/TFA (30/35/35/0.1, all by vol.)

5. Water/acetonitrile/isopropanol/TFA (10/45/45/0.1, all by vol.)

The retained hydrophobic proteins were eluted with $1 \mathrm{~mL}$ $88 \%$ formic acid followed by $2 \mathrm{~mL}$ chloroform $/$ methanol/H2O $(4 / 4 / 1, v / v$, freshly prepared daily). Samples were collected and dried in a vacuum concentrator and stored at $-20^{\circ} \mathrm{C}$. 
Table 2 Number of protein in hydrophobic fraction of 1-mg tissue lysates identified by LTQ-Orbitrap

\begin{tabular}{lccc}
\hline Sample (1mg) & Proteins identified & Sample (1mg) & Proteins identified \\
\hline TNBC \#1 & 130 & Non-TNBC \#1 & 159 \\
TNBC \#2 & 143 & Non-TNBC \#2 & 169 \\
TNBC \#3 & 110 & Non-TNBC \#3 & 127 \\
TNBC \#4 & 94 & Non-TNBC \#4 & 158 \\
TNBC \#5 & 60 & Non-TNBC \#5 & 177 \\
TNBC \#6 & 196 & Non-TNBC \#6 & 116 \\
TNBC \#7 & 154 & Non-TNBC \#7 & 122 \\
TNBC \#8 & 198 & Non-TNBC \#8 \\
TNBC \#9 & 185 & Non-TNBC \#9 & 196 \\
TNBC \#10 & 205 & Non-TNBC \#10 & 178 \\
\hline
\end{tabular}

Reduction, Alkylation, and Trypsinization of Proteins for $\mathrm{LC} / \mathrm{MS} / \mathrm{MS}$

The dried samples were dissolved in freshly prepared guanidine $\mathrm{HCl}(6 \mathrm{M}, 20 \mu \mathrm{l})$ containing $10 \mathrm{mM}$ DTT and $0.2 \%$ RapiGest (Waters, Milford, MA, USA), vortexed, and incubated at $37^{\circ} \mathrm{C}$ for $1 \mathrm{~h}$. Additional guanidine HCL (6 M, $2 \mu \mathrm{l}$ ) containing $300 \mathrm{mM}$ iodoacetamide was added, mixed, and incubated at $37^{\circ} \mathrm{C}$ for $1 \mathrm{~h}$. The sample was digested by trypsin (sequencing grade, Promega) in solution containing $1.6 \mathrm{ml} 0.5 \mathrm{M}$ ammonium bicarbonate and incubated for $4 \mathrm{~h}$ at $37^{\circ} \mathrm{C}$. Reverse phase $\mathrm{C} 18$ cartridges (AccuBond II ODSC18) were used and the manufacturer's protocol was followed to remove salt from the samples.

\section{LC/MS/MS Analysis and Peptide Data Analysis}

LC/MS/MS and data analysis were modified from Whelan et al. [17]. Briefly, samples were redissolved in Buffer A (H2O/acetonitrile/formic acid, 98.9/1/0.1, typically $50 \mu \mathrm{L}$ ), separated by nanospray LC (Eskigent Technologies, Dublin, CA, USA), and analyzed using online tandem mass spectrometry (LTQ-Orbitrap, Thermo Fisher). Aliquots were injected $(10 \mu \mathrm{L})$ onto a reverse phase column (New Objective $\mathrm{C} 18,15 \mathrm{~cm}, 75 \mu \mathrm{M}$ diameter, $5 \mu \mathrm{m}$ particle size equilibrated in Buffer A) and eluted (300 nL/min) with an increasing concentration of Buffer B (acetonitrile/water/ formic acid, 98.9/1/0.1; $\min 0 / 5,10 / 10,112 / 40,130 / 60$, $135 / 90,140 / 90)$. The effluent from the column was passed directly into an integrated nanospray emitter tip connected to the LTQ-Orbitrap mass spectrometer. Eluted peptides were analyzed by MS and data-dependent MS/MS acquisition (collision-induced dissociation (CID)), previously optimized for samples, selecting the seven most abundant precursor ions for MS/MS with a dynamic exclusion duration of $15.0 \mathrm{~s}$.

Biowork software searchers were conducted using a human trypsin cleavage indexed peptide database, with variable modifications of carboxyamidomethylation (57.02146) and methionine oxidation (15.99492). Scaffold data analysis (Proteome Software, Version 2.2.03) was conducted using Bioworks search file results with a high stringency filter with a 99\% minimum protein ID probability, a minimum number of two unique peptides for each protein identified, and with a minimum peptide ID probability of $95 \%$. Scaffold uses X! Tandem [29], ProteinProphet computer algorithms [30], and PeptideProphet [31] to verify peptide identifications derived from MS/MS sequencing results. Scaffold is also used to quantitate spectral counts by normalizing MS/MS data between samples. Each sample analyzed was a combination of three replicate experiments and was normalized by averaging spectral counts for all samples, multiplying spectral counts in each sample by the average and then dividing by each sample's sum.

\section{Western Blotting Analysis}

Twenty micrograms of protein was separated by a $12 \%$ SDSPAGE gel and transferred to a nitrocellulose membrane by electrophoretic blotting. The membrane was blocked with 5\% nonfat dry milk in TBS- $0.1 \%$ Tween 20 and then incubated with primary antibody for $1 \mathrm{~h}$. The blots were then washed 10

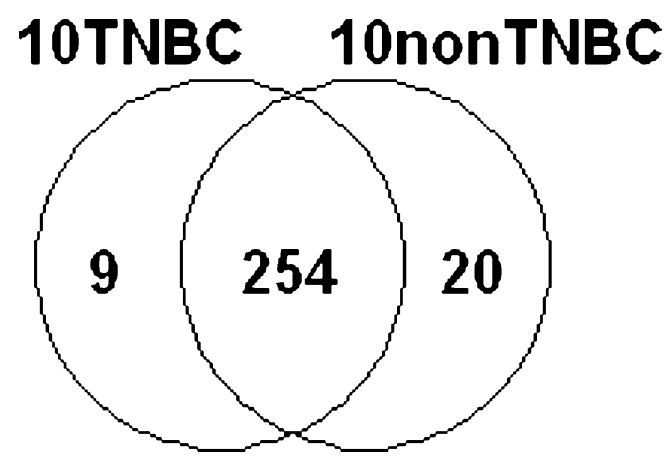

Fig. 1 Many hydrophobic proteins were found to be shared between $\mathrm{TNBC}$ and $\mathrm{ER}+\mathrm{PR}+\mathrm{Her} 2-$ breast cancers (non-TNBC). Unique proteins were also found to be associated with either TNBC or ER+PR+Her2breast cancers. The pool of the unique proteins may include diseaserelated biomarkers, and may potentially be used as therapy targets 
Table 3 Unique proteins detected exclusively in 10 TNBC cases

\begin{tabular}{llc}
\hline Identified proteins & Accession number & Molecular weight (kDa) \\
\hline Cytoskeleton-associated protein 4 & gi|19920317 & 66 \\
HLA class II histocompatibility antigen, DR alpha chain & gi|122206 (+5) & 29 \\
3'-phosphoadenosine 5'-phosphosulfate synthase 1 & gi|46094058 (+1) & 71 \\
HLA class I histocompatibility antigen, A-23 alpha chain & gi|231359 & 41 \\
Transforming growth factor, beta-induced & gi|4507467 & 75 \\
Tapasin isoform 7 & gi|114606885 & 48 \\
Ribosome-binding protein 1 & gi|23822112 & 152 \\
UTP-glucose-1-phosphate uridylyltransferase & gi|2136353 (+1) & 57 \\
Chain A, C-terminal Atpase domain of human Tap1 & gi|15988434 (+1) & 28 \\
\hline
\end{tabular}

times over $30 \mathrm{~min}$ in TBS-Tween 20 and incubated for $1 \mathrm{~h}$ with horseradish peroxidase-conjugated secondary antibody, and then washed in TBS-Tween 20 as before. The membranes were then developed using the Supersignal West Pico Chemiluminescent Western blotting detection system according to the instructions of the manufacturer (Pierce, Arlington Heights, IL, USA). Primary antibodies of keratin 19 and heat shock proteins were purchased from Dako (Carpinteria, CA, USA) and Santa Cruz Biotechnology (Santa Cruz, CA, USA), respectively. Both antibodies were diluted 1:1,000 in Western blot analysis.

\section{Results}

1. Hydrophobic column chromatography combined with the LTQ-Orbitrap MS/MS analysis detected hundreds of proteins from human cancer tissue specimens.
The novel hydrophobic fractionation system effectively enriched hydrophobic proteins of cancer tissues. Combined with the LTQ-Orbitrap, hundreds of proteins were identified in each sample. The significant number of proteins identified provided a meaningful analysis of disease-related biomarkers. Table 2 lists the number of hydrophobic proteins identified by the LTQ-Orbitrap in $1 \mathrm{mg}$ of each of the 20 samples.

2. Many shared and unique proteins were detected in TNBC and non-TNBC specimen.

We compared hydrophobic proteins between 10 cases of TNBC and non-TNBC specimens (Fig. 1 and Tables 3 and 4). As expected, many proteins were found to be shared by TNBC and non-TNBC specimens. However, a significant number of unique proteins were found to be associated with either TNBC or non-TNBC specimens. When confirmed, some proteins unique to TNBC
Table 4 Unique proteins detected exclusively in the 10 $\mathrm{ER}+\mathrm{PR}+\mathrm{Her} 2-$ breast cancer cases

\begin{tabular}{llc}
\hline Identified proteins & Accession number & Molecular weight (kDa) \\
\hline Keratin 18 & gi|4557888 & 48 \\
Perilipin & gi|8134637 & 56 \\
Galectin 3 binding protein & gi| 5031863 & 65 \\
Zinc-alpha-2-glycoprotein & gi|141596 (+1) & 34 \\
Collagen, type VI, alpha 1 & gi|87196339 & 109 \\
Creatine kinase & gi|21536286 & 43 \\
Prostatic binding protein & gi|4505621 (+1) & 21 \\
Vesicle amine transport protein 1 & gi|18379349 & 42 \\
Chain A, cellular retinoic acid binding protein-type Ii & gi|157830381 (+2) & 16 \\
Chain A, N-acetyltransferase 1 F125s mutant & gi|119390179 (+2) & 34 \\
Sodium/hydrogen exchanger regulatory factor 1 & gi|4759140 & 39 \\
FK506 binding protein 52 & gi|4503729 (+1) & 52 \\
Glycerol-3-phosphate dehydrogenase 1 (soluble) & gi|33695088 & 38 \\
Epiplakin 1 & gi|13876386 & 553 \\
Methylcrotonoyl-coenzyme A carboxylase 2 (beta) & gi|11545863 & 61 \\
Keratin 5 & gi|119395754 \\
Chain A, glutamate dehydrogenase-Apo form & gi|20151189 (+3) \\
Phosphofructokinase & gi|48762920 & 62 \\
Chain A, carboxylesterase & gi|114793717 (+1) & 56 \\
\hline
\end{tabular}


may represent valuable biomarkers for developing diagnostics or targeted therapy.

HLA class II histocompatibility antigen DR alpha and HLA class I histocompatibility antigen A-23 alpha are trans-membrane proteins expressed on lymphocytes. More HLA histocompatibility antigens are found in TNBC, indicating a significant presence of lymphocytic infiltration in TNBC specimens, which is a useful histological feature of the TNBC.

3. Differentially expressed proteins by TNBC compared with non-TNBC tissues.

Semi-quantitative analysis of TNBCs vs. non-TNBCs by Scaffold 2.2.03 software showed that numerous proteins were up-regulated $(\geq 2.0$-fold) or downregulated $(\leq 0.5$-fold) in the two types of breast cancer. A list of proteins that up-regulated $(\geq 2.0$-fold changes, Table 5) and down-regulated $(\leq 0.5$-fold changes, Table 6) in TNBC was reported. Our data suggest that these proteins may represent bio-signature of TNBC, which may distinguish TNBC from ER+PR+Her2breast cancers.

4. Data validation by Western blotting analysis.

To confirm the findings observed from mass spectrometry study, we have selectively validated the MS-identified hydrophobic proteins by Western blot (Fig. 2 and data not shown) and found that the higher the spectrum counts of the protein were, the more likely it was that the proteins were identified by both methods. In most cases, the expression levels of each protein detected by Western blot were consistent with the finding of mass spectrometry analysis. The strategy of combing different fractionation methods and LC/MS/MS is useful in the discovery of novel disease-related proteins.

5. Keratins were down-regulated in TNBC.

The keratins have an extraordinary wide presence with 54 functional keratin genes reported in humans, 37

Table 5 Proteins over-expressed by TNBC ( $\geq 2$-fold of up-regulation) when compared with ER+PR+Her2- (non-TNBC) cases

\begin{tabular}{|c|c|c|c|c|}
\hline Proteins over-expressed in TNBC & Accession no. & $\begin{array}{l}\text { Molecular } \\
\text { weight }(\mathrm{kDA})\end{array}$ & Fold of TNBC/non-TNBC & $\begin{array}{l}\text { Average detected } \\
\text { spectra of } \mathrm{TNBC} \pm \mathrm{SE}\end{array}$ \\
\hline $\begin{array}{l}\text { Serine (or cysteine) proteinase inhibitor, } \\
\text { clade } \mathrm{H} \text {, member } 1\end{array}$ & gi|32454741 & 46 & 4.7 & $11.3 \pm 6.4$ \\
\hline Filamin A, alpha isoform 2 & gi|160420317 & 281 & 4.5 & $36.1 \pm 12.9$ \\
\hline Mitochondrial ATP synthase beta subunit & gi|32189394 & 57 & 3.7 & $31.6 \pm 11.1$ \\
\hline Heat shock protein $90 \mathrm{kDa}$ beta, member 1 & gi|4507677 & 92 & 3.3 & $30.1 \pm 8.9$ \\
\hline Tropomyosin 4 isoform 2 & gi|4507651 & 29 & 3.2 & $16.8 \pm 6.6$ \\
\hline Tropomyosin 3 & gi|58652133 & 33 & 3.1 & $1.1 \pm 0.5$ \\
\hline ATP synthase, alpha subunit & gi|4757810 & 60 & 2.9 & $24.8 \pm 6.6$ \\
\hline Heat shock $70 \mathrm{kDa}$ protein 5 & gi|16507237 & 72 & 2.9 & $54.4 \pm 18.2$ \\
\hline $\begin{array}{l}\text { Three-dimensional structure } \\
\text { of a transglutaminase }\end{array}$ & gi|1127268 (+4) & 83 & 2.8 & $6.2 \pm 2.0$ \\
\hline High-mobility group box 1 & gi|4504425 & 25 & 2.7 & $7.8 \pm 2.6$ \\
\hline Moesin & gi|4505257 & 68 & 2.7 & $6.6 \pm 2.8$ \\
\hline Thymidine phosphorylase & gi|118138578 (+1) & 50 & 2.5 & $14.4 \pm 5.6$ \\
\hline Enolase 1 & gi|4503571 & 47 & 2.5 & $53.5 \pm 14.0$ \\
\hline Fascin 1 & gi|4507115 & 55 & 2.3 & $4.2 \pm 2.3$ \\
\hline Fibrinogen, beta chain & gi|70906435 & 56 & 2.3 & $6.0 \pm 3.8$ \\
\hline Ribophorin I precursor & gi|4506675 & 69 & 2.3 & $5.5 \pm 1.9$ \\
\hline Tryptophanyl-tRNA synthetase isoform a & gi|47419914 & 53 & 2.2 & $6.0 \pm 4.2$ \\
\hline $\begin{array}{l}\text { Cytoplasmic domain of erythrocyte } \\
\text { band-3 protein }\end{array}$ & gi|14277739 (+1) & 43 & 2.1 & $6.1 \pm 2.7$ \\
\hline Vimentin & gi|62414289 & 54 & 2.1 & $32.4 \pm 9.6$ \\
\hline Annexin I & gi|4502101 & 39 & 2.1 & $25.7 \pm 11.5$ \\
\hline Vitronectin & gi|88853069 & 54 & 2.1 & $4.4 \pm 1.8$ \\
\hline Lamin $\mathrm{A} / \mathrm{C}$ isoform 1 & gi|27436946 & 74 & 2 & $3.1 \pm 2.2$ \\
\hline Annexin A2 isoform 2 & gi|4757756 & 39 & 2 & $75.6 \pm 20.8$ \\
\hline Collagen, type XII, alpha 1 long isoform & gi|93141047 & 333 & 2 & $2.9 \pm 2.9$ \\
\hline Vitamin D-binding protein I & gi|21730554 (+2) & 42 & 2 & $36.9 \pm 6.7$ \\
\hline Porin 31HM & gi|238427 (+1) & 31 & 2 & $63.7 \pm 1.5$ \\
\hline
\end{tabular}


Table 6 Selected proteins down-regulated $(\leq 0.5$-fold) in triple-negative breast cancer (TNBC) tumor when compared with ER+PR+Her2- (nonTNBC) tumor specimens (keratin data are listed in Table 7)

\begin{tabular}{|c|c|c|c|c|}
\hline Proteins down-regulated in TNBC & Accession no. & $\begin{array}{l}\text { Molecular } \\
\text { weight }(\mathrm{kDa})\end{array}$ & Fold of TNBC/non-TNBC & $\begin{array}{l}\text { Average detected } \\
\text { spectra of } \mathrm{TNBC} \pm \mathrm{SE}\end{array}$ \\
\hline Fatty acid synthase & gi|67476453 & 273 & 0.08 & $1.0 \pm 0.7$ \\
\hline Collagen alpha-3(VI) chain & gi|5921193 & 344 & 0.2 & $5.8 \pm 1.9$ \\
\hline Amine oxidase, copper containing 3 & gi|4502119 (+1) & 85 & 0.3 & $1.1 \pm 0.5$ \\
\hline UDP-glucose dehydrogenase & gi|4507813 & 55 & 0.4 & $0.1 \pm 0.1$ \\
\hline Collagen, type XIV, alpha 1 & gi|55743096 & 194 & 0.4 & $13.6 \pm 3.7$ \\
\hline Triosephosphate isomerase 1 isoform 1 & gi|4507645 (+2) & 27 & 0.5 & $6.5 \pm 1.9$ \\
\hline Periostin, osteoblast specific factor isoform 1 & gi|209862907 & 93 & 0.5 & $7.4 \pm 2.8$ \\
\hline Transketolase isoform 1 & gi|4507521 & 68 & 0.5 & $9.2 \pm 4.3$ \\
\hline Heat shock protein beta-1 & gi|4504517 & 23 & 0.5 & $1.0 \pm 0.6$ \\
\hline Lumican precursor & gi|4505047 & 38 & 0.5 & $5.8 \pm 2.4$ \\
\hline Plasma membrane associated protein, S3-12 & gi|122937195 & 134 & 0.5 & $0.5 \pm 0.4$ \\
\hline Osteoglycin & gi|7661704 & 34 & 0.5 & $0.5 \pm 0.3$ \\
\hline Immunoglobulin $\mathrm{J}$ chain & gi|400044 & 16 & 0.5 & $0.4 \pm 0.2$ \\
\hline $\begin{array}{l}\text { Chain A, the intact and cleaved human } \\
\text { antithrombin Iii complex }\end{array}$ & gi|999513 & 49 & 0.5 & $3.7 \pm 2.4$ \\
\hline CD36 antigen & gi|48375176 & 53 & 0.5 & $0.5 \pm 0.5$ \\
\hline Zinc-alpha-2-glycoprotein & gi|141596 (+1) & 34 & 0.5 & $0.1 \pm 0.1$ \\
\hline Perilipin & gi|8134637 & 60 & 0.5 & $0.1 \pm 0.1$ \\
\hline
\end{tabular}

of which are epithelial keratins. Keratins are classified according to their isoelectric points; type I is acidic and type II is basic or neutral. Keratins are incorporated into filaments by heteropolymeric pair formation of type I and type II (1:1) molecules [32-34]. Keratins are not only important for the mechanical stability and integrity of epithelial cells and tissues [32]. Moreover, some keratins also have regulatory functions and are involved in intracellular signaling pathways, e.g., protection from stress, wound healing, and apoptosis [32].

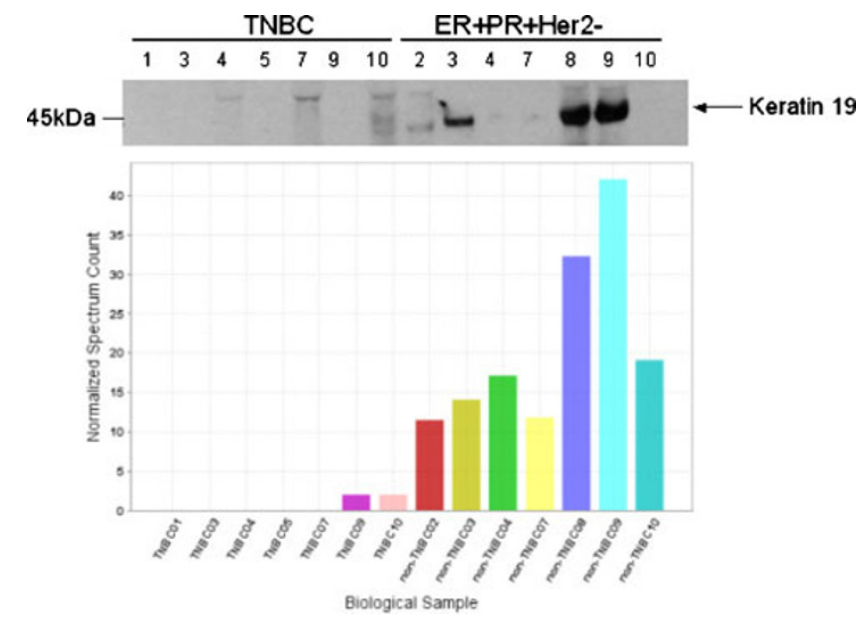

Fig. 2 Mass data of keratin 19 expression in 14 breast cancer cases are consistent with Western blot analysis
Mass spectrometric analysis of the hydrophobic sub-proteome showed nine different epithelial keratins (Table 7), all of which are over-expressed in the $\mathrm{ER}+\mathrm{PR}+\mathrm{Her} 2-$ breast cancer than the TNBC. This finding has been confirmed by Western blot analysis with keratin 19 expression in 14 breast cancer cases (Fig. 2).

6. Heat shock proteins were up-regulated in TNBC.

Heat shock proteins (Hsp90, Hsp70) are chaperones that assist the proteins in their folding, stability, assembly into multi-protein complexes, and transportation across cellular membranes. The expression of heat shock proteins is highly inducible by a wide variety of physiological and environmental stresses [35]. Heat shock proteins have cyto-protective functions by anti-apoptosis to promote the survival of stressed cells. In TNBC cancer tissues, Hsp70 and Hsp90 were found to be expressed consistently at higher level than that of $\mathrm{ER}+\mathrm{PR}+\mathrm{Her} 2$ breast cancer (Table 8 ). The results have been confirmed by Western blot analysis (data not shown).

\section{Discussion}

In this study, we have investigated the differences of hydrophobic sub-proteomes between two subtypes of breast cancers (TNBC vs. ER+PR+Her2-). Mass spectrometry (LC/MS/MS) techniques were used to compare the protein 
Table 7 Epithelial keratins detected in specimens of $10 \mathrm{TNBC}$ and $10 \mathrm{ER}+\mathrm{PR}+\mathrm{Her} 2-$ (non-TNBC) breast cancer

\begin{tabular}{lllccc}
\hline $\begin{array}{l}\text { Identified epithelial keratin } \\
\text { (new nomenclature) }\end{array}$ & Type & Accession number & Molecular weight (kDa) & Ratio of non-TNBC/TNBC & $\begin{array}{l}\text { Average detected spectra } \\
\text { of TNBC } \pm \text { SE }\end{array}$ \\
\hline Keratin 1 (K1) & II & gi|1346343 & 66 & 2.5 & $16.3 \pm 4.0$ \\
Keratin 2 (K2) & II & gi|547754 & 66 & 2.0 & $3.3 \pm 1.1$ \\
Keratin 5 (K5) & II & gi|119395754 & 62 & 2.0 & $1.0 \pm 0.0$ \\
Keratin 7 (K7) & II & gi|20178293 & 51 & 3.3 & $1.6 \pm 1.2$ \\
Keratin 8 (K8) & II & gi|4504919 & 54 & 3.3 & $0.4 \pm 0.3$ \\
Keratin 9 (K9) & I & gi| 81175178 & 62 & 10.0 & $1.1 \pm 1.1$ \\
Keratin 10(K10) & I & gi| 147744568 & 60 & 5.0 & $6.7 \pm 2.0$ \\
Keratin 18 (K18) & I & gi|4557888 & 48 & 2.5 & $0.2 \pm 0.2$ \\
Keratin 19 (K19) & I & gi| 90111766 & 44 & 3.3 & $2.3 \pm 1.3$ \\
\hline
\end{tabular}

profiles identified from the 20 tumors, with 10 in each of the comparison groups. We also report several candidate proteins that may be involved in the aggressive manifestation of TNBC.

An interesting finding of this study was to discover that all nine keratins found in breast cancers were downregulated in all 10 TNBC cases when compared with the 10 ER+PR+Her2- breast cancer cases. Keratins (previously called cytokeratins) are filament-forming proteins and are essential for normal tissue structure and function [36]. A major function of epithelial keratins is to provide the mechanical stability and integrity of epithelial cells and tissues [32], as well as the mechanical support to epithelial cell sheets [37]. The biological meaning and mechanism of reduced keratins in TNBC has not been previously investigated. Hypothetically, however, less keratin may indicate that TNBC cells are less "stiff" and more "flexible" in changing shapes to facilitate cellular movement, transportation, and migration for its invasive nature and early metastasis. It has been reported that metastatic cancer cells are $70 \%$ softer than non-metastatic cells using nanomechanical analysis [38]. Down-regulated expression of keratins in TNBC was also observed by another study in our laboratory (Whelan et al., submitted for publication), which compares TNBC with Her2+ breast cancers. In this study, keratin 19 was found expressed eightfold lower in TNBC. Thus, change in cell stiffness may be a newly described characteristic of cancer cells that affects the way they spread and the molecules regulate this function.

Annexin family proteins were found over-expressed in TNBC (Table 5), which is consistent with our previous finding $[14,19]$ in TNBC tumors and cell lines. The protein family of annexins has continued to grow since their association with intracellular membranes was first reported in 1977 [39]. Annexins are important in various cellular and physiological functions such as providing a membrane scaffold, which is relevant to changes in the cell's shape $[40,41]$. Also, annexins have been shown to be involved in trafficking and organization of vesicles, exocytosis, endocytosis, and calcium ion channel formation [42]. A literature search indicates that Annexin over-expression correlates with the aggressiveness of cancer. Annexin A3 was significantly up-regulated in invasive lung adenocarcinomas with lymph node metastasis compared to those without lymph node metastasis [43]. Similarly, annexin A5 and A7 are significantly elevated in lymphatic metastasis of mouse hepatocarcinoma [44]. Together, these findings provide strong evidence that Annexin family proteins are
Table 8 Selected heat shock proteins detected in specimens of $10 \mathrm{TNBC}$ and $10 \mathrm{ER}+\mathrm{PR}+\mathrm{Her} 2-($ non-TNBC) breast cancer cases

\begin{tabular}{|c|c|c|c|}
\hline Identified heat shock proteins & Accession number & $\begin{array}{l}\text { Fold change of } \\
\text { TNBC/non-TNBC }\end{array}$ & $\begin{array}{l}\text { Average detected } \\
\text { spectra of } \\
\mathrm{TNBC} \pm \mathrm{SE}\end{array}$ \\
\hline Heat shock $70 \mathrm{kDa}$ protein 5 & gi|16507237 & 2.9 & $54.4 \pm 18.2$ \\
\hline Heat shock $90 \mathrm{kDa}$ protein 1 , beta & gi|20149594 & 1.1 & $30.7 \pm 7.6$ \\
\hline Heat shock $70 \mathrm{kDa}$ protein 8 isoform 1 & gi|5729877 & 1.4 & $24.7 \pm 8.8$ \\
\hline $\begin{array}{l}\text { Heat shock protein } 90 \mathrm{kDa} \text { beta, } \\
\text { member } 1\end{array}$ & gi|4507677 & 3.3 & $30.1 \pm 8.9$ \\
\hline Heat shock $70 \mathrm{kDa}$ protein 9 precursor & gi| 24234688 & $\begin{array}{l}1.8 \\
\text { Average fold }=2.1\end{array}$ & $8.6 \pm 3.8$ \\
\hline
\end{tabular}


likely to contribute to the aggressive phenotype and metastatic potential of TNBC.

In this study, we also found an overall up-regulation of heat shock protein expression in TNBC. Heat shock proteins are a class of functionally related proteins whose expression is transcriptionally regulated and over-expressed when cells are exposed to elevated temperatures, hypoxia, or other stress [45]. Heat shock proteins are named according to their molecular weight. Among them, Hsp60, Hsp70, and Hsp90 are the most widely studied Hsps. Hsps have a dual function depending on their intracellular or extracellular location. The different properties of Hsps allow them to be exploited in therapy. Intracellular Hsps are protective to the cell and are highly expressed in cancerous cells. The elevated Hsp expression has been shown to promote cancer growth through inhibition of programmed cell death (Hsp27, Hsp70), allowance of autonomous tumor growth (Hsp90) and tumor resistance to chemotherapy and hyperthermia [46]. Since they participate in oncogenesis and in resistance to treatment, the inhibition of Hsps has been tested in clinical trial for cancer treatment. Small molecule inhibitors of Hsps, especially Hsp90, show promise as anticancer agents [35]. The potent Hsp90 inhibitor 17-AAG is currently in clinical trials for the treatment of several types of cancer [47]. In contrast to the intracellular Hsps, extracellularly located or membranebound Hsps mediate immunological functions. They can elicit an immune response modulated either by the adaptive or innate immune system [35]. Their immunogenicity can be used to form the basis of anticancer vaccines [46].

Our mass spectrometric results showed a significant over-expression of Hsp70 and Hsp90 in the TNBC tumors. Another parallel study comparing TNBC with Her2+ tumors in our laboratory indicates that Hsp70 and Hsp90 are over-expressed in TNBC (He et al., in preparation). This is consistent with other studies reporting Hsp70 and Hsp90 expression associated with tumor of poor differentiation, rapid proliferation, and suppressed apoptosis. Clinically, they are associated with lymph node metastasis and poor clinical outcome [48-50].

Among the nine unique proteins detected exclusively in 10 TNBC cases, two of them were HLA class proteins (Table 3): HLA class II histocompatibility antigen DR alpha and HLA class I histocompatibility antigen A-23 alpha were both over-expressed in TNBC but below detection level in $\mathrm{ER}+\mathrm{PR}+\mathrm{Her} 2-$ breast cancer specimens. These two proteins belong to the major histocompatibility complex (MHC) class II and I family, respectively. They are membrane proteins, which explain the reason they exist in hydrophobic fractions.

The MHC is a large gene family, which is the most genedense region of the mammalian genome and plays an important role in the immune system and autoimmunity
[51]. The MHC proteins display both self antigens (peptide fragments from the cell itself) and non-self antigens (e.g., fragments of invading microorganisms or cancer) to $\mathrm{T}$ cells and NK cells. Under normal conditions, expression of MHC molecules inhibits killing by NK cells, while absence of MHC molecules in infected or stressed cells allows cell-mediated cytotoxicity to occur. MHC class I molecules express on all nucleated cells, while MHC class II family proteins express on most immune system cells, specifically on antigen-presenting cells. Thus, over-expression of MHC class II and I proteins in tumor tissue suggests two possibilities. First, antigens may exist on tumor cell surfaces carrying different biological functions to tumor cells. Second, TNBC may have more lymphocytic infiltration, which may be the source of MHC over-expression found in these tumors. Recent studies reported that several HLA protein over-expression are associated with breast cancer $[52,53]$ and may favor their escape from anti-tumor immune responses [54], as well as predict chemotherapy resistance [55].

The rapid development of analytical instruments and data mining software in the last two decades has significantly extended the possibilities of studying proteins in life sciences. Proteomic analysis provides deeper insights into qualitative and quantitative changes in protein composition in association with the disease process. The combination of hydrophobic fractionation and nano-LC mass spectrometry analysis of cancer proteome offers a promising tool in the discovery of potential cancer bio-signatures. When sufficiently refined, this approach may prove useful for early detection and better treatment of breast cancer.

Acknowledgments Grant support: The present study was supported by grant funds from NIH (NCI \#1RO1 CA 093736-01A1), the Gonda Foundation, Entertainment Industry Foundation (EIF)/Women's Cancer Research Fund, and Friends of the Breast Program at UCLA.

Disclosure of Potential Conflicts of Interest No potential conflicts of interest were disclosed.

Open Access This article is distributed under the terms of the Creative Commons Attribution Noncommercial License which permits any noncommercial use, distribution, and reproduction in any medium, provided the original author(s) and source are credited.

\section{References}

1. Jemal A, Siegel R, Ward E, Hao Y, Xu J, Thun MJ. Cancer statistics, 2009. CA Cancer J Clin. 2009;59:225-49.

2. Cleator S, Heller W, Coombes RC. Triple-negative breast cancer: therapeutic options. Lancet Oncol. 2007;8:235-44.

3. Kang SP, Martel M, Harris LN. Triple negative breast cancer: current understanding of biology and treatment options. Curr Opin Obstet Gynecol. 2008;20:40-6.

4. Perou CM, Sørlie T, Eisen MB, et al. Molecular portraits of human breast tumours. Nature. 2000;406:747-52. 
5. Sorlie T, Tibshirani R, Parker J, et al. Repeated observation of breast tumor subtypes in independent gene expression data sets. Proc Natl Acad Sci USA. 2003;100:8418-23.

6. Nielsen TO, Hsu FD, Jensen K, et al. Immunohistochemical and clinical characterization of the basal-like subtype of invasive breast carcinoma. Clin Cancer Res. 2004;10:5367-74.

7. Harris LN, You F, Schnitt SJ, et al. Predictors of resistance to preoperative trastuzumab and vinorelbine for HER2-positive early breast cancer. Clin Cancer Res. 2007;13:1198-207.

8. Carey LA, Perou CM, Livasy CA, et al. Race, breast cancer subtypes, and survival in the Carolina Breast Cancer Study. JAMA. 2006;295:2492-502.

9. Bauer KR, Brown M, Cress RD, et al. Descriptive analysis of estrogen receptor (ER)-negative, progesterone receptor (PR)negative, and HER2-negative invasive breast cancer, the socalled triple-negative phenotype: a populationbased study from the California cancer Registry. Cancer. 2007;109:1721-8.

10. Dawson SJ, Provenzano E, Caldas C. Triple negative breast cancers: clinical and prognostic implications. Eur J Cancer. 2009;45 Suppl 1:27-40.

11. Furr BJ, Jordan VC. The pharmacology and clinical uses of tamoxifen. Pharmacol Ther. 1984;25:127-205.

12. Dent R, Trudeau M, Pritchard KI, et al. Triple-Negative Breast Cancer: Clinical Features and Patterns of Recurrence. Clin Cancer Res. 2007;13:4429-34.

13. Jordan VC. Tamoxifen (ICI46, 474) as a targeted therapy to treat and prevent breast cancer. Br J Pharmacol. 2006;147 Suppl 1: S269-76.

14. He J, Gornbein J, Shen D, et al. Detection of breast cancer biomarkers in nipple aspirate fluid by SELDI-TOF and their identification by combined liquid chromatography-tandem mass spectrometry. Int J Oncol. 2007;30:145-54.

15. Shau H, Chandler GS, Whitelegge JP, Gornbein JA, Faull KF, Chang HR. Proteomic profiling of cancer biomarkers. Brief Funct Genomic Proteomic. 2003;2:147-58.

16. He J, Shen D, Chung DU, et al. Tumor proteomic profiling predicts the susceptibility of breast cancer to chemotherapy. Int $\mathrm{J}$ Oncol. 2009;35:683-92.

17. Whelan SA, Lu M, He J, et al. Mass spectrometry (LC-MS/MS) site-mapping of N-glycosylated membrane proteins for breast cancer biomarkers. J Proteome Res. 2009;8:4151-60.

18. Lu M, Faull KF, Whitelegge JP, Saxton RK, Shen D, Chang HR. Mass spectrometry for cancer biomarker discovery. Biomark Insights. 2007;2:347-60.

19. Lu M, Whitelegge JP, Whelan SA, et al. Hydrophobic fractionation enhances novel protein detection by mass spectrometry in triple negative breast cancer. J Proteomics Bioinform. 2010;3:1-10.

20. Hirokawa T, Boon-Chieng S, Mitaku S. SOSUI: classification and secondary structure prediction system for membrane proteins. Bioinformatics. 1998;14:378-9.

21. Hopkins AL, Groom CR. The druggable genome. Nat Rev Drug Discov. 2002;1:727-30.

22. Speers AE, Wu CC. Proteomics of integral membrane proteinstheory and application. Chem Rev. 2007;107:3687-714.

23. Whitelegge JP, Halgand F, Souda P, Zabrouskov V. Top-down mass spectrometry of integral membrane proteins. Expert Rev Proteomics. 2006;3:585-96.

24. Whitelegge JP. Mass spectrometry for high throughput quantitative proteomics in plant research: lessons from thylakoid membranes. Plant Physiol Biochem. 2005;42:919-27.

25. Whitelegge JP, Gunderson CB, Faulk KF. Electrospray-ionization mass spectrometry of intact intrinsic membrane proteins. Protein Sci. 1998;7:1423-30

26. Whitelegge JP, Katz J, Pihakari K, et al. Subtle modification of isotope ratio proteomics (SMIRP); a new strategy for expression proteomics. Phytochemistry. 2004;65:1507-15.
27. Whitelegge JP, Le Coutre J, Lee JC, et al. Towards the bilayer proteome electrospray ionization mass spectrometry of large intact membrane proteins. Proc Natl Acad Sci. 1999;96:10695-8.

28. Whitelegge JP. Tandem mass spectrometry of integral membrane proteins for top-down proteomics. Trends Anal Chem. 2005;24:576-82.

29. Craig R, Beavis RC. A method for reducing the time required to match protein sequences with tandem mass spectra. Rapid Commun Mass Spectrom. 2003;17:2310-16.

30. Nesvizhskii AI, Keller A, Kolker E, Aebersold R. A statistical model for identifying proteins by tandem mass spectrometry. Anal Chem. 2003;75:4646-58.

31. Keller A, Nesvizhskii AI, Kolker E, Aebersold R. Empirical statistical model to estimate the accuracy of peptide identifications made by MS/MS and database search. Anal Chem. 2002;74:5383-92.

32. Moll R, Divo M, Langbein L. The human keratins: biology and pathology. Histochem Cell Biol. 2008;129:705-33.

33. Tseng SC, Jarvinen MJ, Nelson WG, Huang JW, WoodcockMitchell J, Sun TT. Correlation of specific keratins with different types of epithelial differentiation: monoclonal antibody studies. Cell. 1982;30:361-72.

34. Wu YJ, Parker LM, Binder NE, et al. The mesothelial keratins: a new family of cytoskeletal proteins identified in cultured mesothelial cells and nonkeratinizing epithelia. Cell. 1982;31:693-703.

35. Didelot C, Lanneau D, Brunet M, et al. Anti-cancer therapeutic approaches based on intracellular and extracellular heat shock proteins. Curr Med Chem. 2007;14:2839-47.

36. Schweizer J, Bowden PE, Coulombe PA, et al. New consensus nomenclature for mammalian keratins. J Cell Biol. 2006;174:169-74.

37. Ma L, Xu J, Coulombe PA, Wirtz D. Keratin filament suspensions show unique micromechanical properties. J Biol Chem. 1999;274:19145-51.

38. Cross SE, Jin YS, Rao J, Gimzewski JK. Nanomechanical analysis of cells from cancer patients. Nat Nanotechnol. 2007;2:780-3.

39. Donnelly SR, Moss SE. Annexins in the secretory pathway. Cell Mol Life Sci. 1997;53:533-8.

40. Creutz CE, Pazoles CJ, Pollard HB. Identification and purification of an adrenal medullary protein (synexin) that causes calciumdependent aggregation of isolated chromaffin granules. J Biol Chem. 1978;253:2858-66.

41. Kenis H, van Genderen H, Bennaghmouch A, et al. Cell surfaceexpressed phosphatidylserine and annexin A5 open a novel portal of cell entry. J Biol Chem. 2004;279:52623-9.

42. Gerke V, Creutz CE, Moss SE. Annexins: linking Ca2+ signalling to membrane dynamics. Nat Rev Mol Cell Biol. 2005;6:449-61.

43. Liu Y, Xiao Z, Li M, et al. Quantitative proteome analysis reveals annexin $\mathrm{A} 3$ as a novel biomarker in lung adenocarcinoma. J Pathol. 2009;106:570-9.

44. Liu S, Sun MZ, Tang JW, Wang Z, Sun C, Greenaway FT. Highperformance liquid chromatography/nanoelectrospray ionization tandem mass spectrometry, two-dimensional difference in-gel electrophoresis and gene microarray identification of lymphatic metastasis-associated biomarkers. Rapid Commun Mass Spectrom. 2008;22:3172-8.

45. De Maio A. Heat shock proteins: facts, thoughts, and dreams. Shock. 1999;11(1):1-12.

46. Calderwood SK, Ciocca DR. Heat shock proteins: stress proteins with Janus-like properties in cancer. Int J Hyperthermia. 2008;24:31-9.

47. Solit DB, Rosen N. Hsp90: a novel target for cancer therapy. Curr Top Med Chem. 2006;6:1205-14.

48. Ciocca DR, Calderwood SK. Heat shock proteins in cancer: diagnostic, prognostic, predictive, and treatment implications. Cell Stress Chaperones. 2005;10:86-103.

49. Jaattela M. Escaping Cell Death: Survival Proteins in Cancer. Exp Cell Res. 1999;248:30-43. 
50. Mosser DD, Caron AW, Bourget L, et al. The chaperone function of hsp70 is required for protection against stress-induced apoptosis. Mol Cell Biol. 2000;20:7146-59.

51. MHC Sequencing Consortium. Complete sequence and gene map of a human major histocompatibility complex. Nature. 1999;401:921-3.

52. Leong PP, Muhammad R, Ibrahim N, Cheong SK, Seow HF. HLA-A and breast cancer in West Peninsular Malaysia. Med Oncol 2010; in press.

53. He X, Dong DD, Yie SM, et al. HLA-G expression in human breast cancer: implications for diagnosis and prognosis, and effect on allocytotoxic lymphocyte response after hormone treatment in vitro. Ann Surg Oncol. 2010;17:145969.

54. Sayed D, Badr G, Maximous D, Mikhail NN, Abu-Tarboush F, Alhazza IM. HLA-G and its relation to proliferation index in detection and monitoring breast cancer patients. Tissue Antigens. 2010;75:40-7.

55. de Kruijf EM, van Nes JG, Sajet A, et al. The predictive value of HLA class I tumor cell expression and presence of intratumoral Tregs for chemotherapy in patients with early breast cancer. Clin Cancer Res. 2010;16:1272-80. 